\title{
Exploring Multimodal Ocular Imaging for Retinal Biomarkers of Alzheimer's Disease, Frontotemporal Dementia, and Cognitively Normal Subjects
}

\section{Kelly Z. Young}

University of Michigan Medicine: University of Michigan Michigan Medicine

Nikhila S. Khandwala

Illinois Eye and Ear Infirmary

Omar Moinuddin

University of Michigan Medicine: University of Michigan Michigan Medicine

Benjamin K. Young

University of Michigan Medicine: University of Michigan Michigan Medicine

Warren Pan

University of Michigan Medicine: University of Michigan Michigan Medicine

\section{Sanjana K. Sathrasala}

University of Michigan Medicine: University of Michigan Michigan Medicine

Yunshu Zhou

University of Michigan Medicine: University of Michigan Michigan Medicine

Kerby Shedden

University of Michigan Medicine: University of Michigan Michigan Medicine

Robert Koeppe

University of Michigan Medicine: University of Michigan Michigan Medicine

\section{Sami J. Barmada}

University of Michigan Medicine: University of Michigan Michigan Medicine

Roger L. Albin

University of Michigan Medicine: University of Michigan Michigan Medicine

Cagri G. Besirli ( $\boldsymbol{D}$ cbesirli@med.umich.edu )

University of Michigan Medicine: University of Michigan Michigan Medicine

\section{Research}

Keywords: Alzheimer disease, Frontotemporal dementia, ocular coherence tomography, ocular imaging, retina

Posted Date: August 18th, 2021 
DOl: https://doi.org/10.21203/rs.3.rs-806032/v1

License: (c) (1) This work is licensed under a Creative Commons Attribution 4.0 International License. Read Full License 


\section{Abstract}

Background and Objectives

Alzheimer's disease (AD) and frontotemporal dementia (FTD) are difficult to reliably differentiate clinically. While their distinct pathologies may be captured by existing diagnostic modalities, these are expensive, time-consuming, and often inaccessible. Dementias are associated with visual dysfunctions, perhaps due to changes in the retina, a developmental outgrowth of the central nervous system. We explore the role of multimodal ocular imaging in the diagnosis of two dementias, AD and FTD.

\section{Methods}

We recruited 5 AD participants, 2 FTD participants, and 9 age-matched controls. Each participant underwent comprehensive ophthalmologic examination and imaging (optical coherence tomography (OCT), OCT-angiography (OCTA), wide-field fundus photography, near-infrared imaging, and fundus autofluorescence). Ocular findings were correlated with cerebral amyloid burden, as measured by $\left[{ }^{11} \mathrm{C}\right] \mathrm{PiB}$ PET.

Results

OCT analysis identified a trend toward differences in retinal nerve fiber layer (RNFL) thicknesses among dementia subtypes $(p=0.064)$. AD eyes had increased RNFL thicknesses compared to FTD $(p=0.046)$ and control eyes $(p=0.046)$, and AD RNFL thickness was positively associated with amyloid burden $(p=$ 0.037). OCTA fractal analysis revealed decreased vascular complexity within the retinal superficial vascular complex in AD compared to FTD eyes $(p=0.035)$. Lastly, fundus autofluorescence demonstrated increased signal intensity in $A D$ eyes compared to control eyes $(p=0.046)$. However, these findings were not statistically significant following correction for multiple comparisons.

Discussion

Despite identifying trends toward differences in retinal layer thicknesses unique to individuals with $A D$ and FTD, our study suggests that changes in retinal thicknesses alone may not serve as reliable biomarkers for distinguishing between dementia subtypes. However, multiple ocular imaging modalities may be considered in conjunction with clinical presentation to aid in diagnosing and monitoring $A D$ and FTD.

Trial registration

The study was prospectively registered on clinicaltrials.gov (NCT03699644) on October 9, 2018.

\section{Introduction}


Alzheimer's disease (AD) and frontotemporal dementia (FTD) exhibit distinct pathologies.(1) However, these conditions may be difficult to distinguish from one another ante mortem, hampering treatment recommendations and clinical trial enrollment. Detailed neuropsychiatric evaluation, cerebrospinal fluid (CSF) analysis, and anatomic or functional imaging all improve diagnostic accuracy. However, such modalities are expensive, time-consuming, often inaccessible, and subject to bias.

The retina is a developmental outgrowth of the brain, and visual dysfunction is well-documented in dementia patients ${ }^{1}$. Given advancements in ocular imaging techniques, the retina is promising for investigating the pathogenesis, clinical course, and diagnosis of dementia. Optical coherence tomography (OCT) is an established non-invasive technique to obtain high-resolution, three-dimensional, cross sectional images of the retina by capturing the intensity and time delay of backscattered light from internal retinal microstructures(2). OCT has been used to evaluate and discriminate among retinal anatomical changes associated with a variety of neuropathologies, including multiple sclerosis, Parkinson's disease, and neuromyelitis optica(3-5).

OCT analysis also shows promise in differentiating between different dementia subtypes. Using OCT, multiple studies have demonstrated significant decreases in retinal nerve fiber layer (RNFL) thickness in $A D$ eyes compared to controls(6). Additional investigations attempted to correlate cognitive function test scores with RNFL thickness or use retinal imaging to diagnose patients with suspected $A D(7)$. While relatively few in number, studies in FTD eyes suggest reduced RNFL thickness, with a proportional relationship between RNFL thickness and Mini Mental Status Exam (MMSE) scores(1). Other investigators observed specific thinning of the outer retinal layers in FTD eyes $(8,9)$. This selective thinning of the outer nuclear layer $(\mathrm{ONL})$ and ellipsoid zone is in contrast to the described preferential RNFL thinning observed in those with $A D(1)$. However, few studies have directly compared retinal structural findings in $A D$ and FTD, with only one report to our knowledge describing similar retinal neuroaxonal thinning in both AD and FTD eyes(1).

Separate from changes in RNFL thickness, ocular imaging tools can also be employed to identify pathophysiological events unique to specific dementias, such as intrinsically fluorescent amyloid plaques in $A D(10)$. Koronyo et al. visualized retinal $A \beta$ plaques using curcumin, a fluorescent ligand that binds fibrillar $A \beta$, in retinas of postmortem $A D$ patients, retinas from mouse models of $A D$, and retinas from living $A D$ patients $(11,12)$. Additionally, Snyder et al. reported a trend toward larger surface areas of $A \beta-$ rich inclusion bodies identified with BluePeak autofluorescence imaging in a population of $A \beta$-positive patients confirmed by neocortical amyloid aggregation (florbetapir PET imaging)(13). On the other hand, autofluorescent lipofuscin has been detected in the retinas of FTD patients and in the eyes of animal models of dementia(14). These studies suggest the potential for in vivo identification of amyloid fibrils and other dementia-specific proteinaceous deposits in the retina, supporting the prospect of identifying retinal protein biomarkers of dementia.

Recent emphasis has been placed on understanding vascular contributions to dementia. Accumulating evidence suggests alterations in retinal vasculature and retinal blood flow in $A D, M C l$, and other forms of 
dementia(7). Ocular imaging provides a promising non-invasive approach to visualizing and investigating retinal vasculature in vivo. For example, several groups report increased area of the foveal avascular zone (FAZ) in AD eyes compared to cognitively normal control ( $C N$ ) eyes, which may reflect vasoconstriction and capillary loss $(15,16)$. Additional studies have also proposed differences in vessel branching, tortuosity, and density in $A D(15,17-21)$ and blood flow and pulsatility $(22,23)$.

Prior ocular imaging studies have been limited by small sample sizes, reliance on clinical diagnoses and differentiation of dementias, presence of confounding ocular diseases, and presence of confounding agerelated retinal changes $(1,24)$. Improved characterization of dementia subjects is necessary to determine whether OCT imaging may identify reliable non-invasive retinal biomarkers of specific dementia subtypes. Our study aims to investigate structural, protein-related, and vascular retinal changes using a comprehensive retinal imaging approach in biomarker confirmed (PET amyloid-positive) AD subjects, amyloid-negative FTD subjects, and amyloid-negative $\mathrm{CN}$ control subjects. Furthermore, we compare ocular findings between eyes from two pathologically distinct dementias, AD and FTD, to identify dementia-specific ocular biomarkers that may improve non-invasive diagnosis and differentiation of dementia subtypes.

\section{Methods}

\section{Standard Protocol Approvals, Registrations, and Patient Consents}

This is a case-control study approved by the Institutional Review Board at the University of Michigan. The study was registered on clinicaltrials.gov (NCT03699644) on October 9, 2018. All imaging studies were acquired from 7 patient participants ( $n=5 A D ; n=2$ FTD) fulfilling diagnostic criteria and 9 age-matched CN controls. Participants were recruited between 2018 to 2019. Written informed patient consent was received by all participants who were able to provide consent. For patients with advanced dementia, assents were obtained from the study participants and written informed consent was obtained by their legally authorized representatives.

\section{Inclusion and Exclusion Criteria}

Subjects were included in the study if they were between the ages of 45 and 85 with a known diagnosis of moderate to severe AD or FTD, as defined by a documented Montreal Cognitive Assessment (MoCA) or Mini-Mental State Exam (MMSE) score of $<17$ and clinical diagnosis defined by ICD 9 and ICD 10. CN age-matched controls were included if they were between the ages of 45 and 80 with no prior evidence of AD or FTD. Subjects were excluded from the study if they had prior surgical enucleation, known preexisting retinal or optic nerve disorders, known corneal eye disease, currently taking ethambutol or hydroxychloroquine, were pregnant or lactating, were unable to be independently and reliably positioned for ocular imaging, had implants or hardware that would interfere with imaging, had pacemaker or defibrillator devices, previously had a stroke, severe heart disease, brain cancer, participating in another 
clinical study which involves usage of an experimental drug or clinical device, were currently undergoing chemotherapy or radiation, had severe high blood pressure, severe diabetes, anemia, liver disease, kidney disease, amyloidosis, Parkinson's disease, or other significant or unstable health conditions that may have precluded safe participation.

\section{Ophthalmological Examination and Imaging}

At the initial study visit, all subjects underwent comprehensive ophthalmological examination at the University of Michigan Kellogg Clinical Research Center, including assessment of visual acuity, refraction, slit lamp examination, dilated fundus examination, and applanation tonometry. All subjects participated in the following forms of ocular imaging: wide-field fundus photography, infrared reflectance (Heidelberg), fundus autofluorescence (Heidelberg), spectral-domain optical coherence tomography (SD-OCT) (Heidelberg), and OCT-angiography (OCTA) (Heidelberg). All images were taken with Heidelberg HRA + OCT2, Heidelberg FA + OCT, and Heidelberg OCT2.

Peripapillary Retinal Nerve Fiber Layer. Peripapillary retinal nerve fiber layer (RNFL) measurements were automatically segmented by native Heidelberg software on OCT Radial Circle Scans (Fig. 1A; Heidelberg Engineering v6.12, Heidelberg Germany). OCT imaging was centered on the optic nerve and the mean peripapillary RNFL thicknesses (Fig. 1A) were measured globally and in the following regions: nasal, inferonasal, supranasal, temporal, inferotemporal, and supratemporal using a $3.5 \mathrm{~mm}$ diameter setting (Fig. 1B).

Macular Thickness Maps. Thickness maps and measurements were automatically generated using native Heidelberg software on OCT Volume Scans using a 1, 3, 6 mm grid type (Heidelberg Engineering v6.12, Heidelberg Germany; Fig S1A). Images were centered on the fovea and measurements were taken between the internal limiting membrane (ILM) and the Bruch's membrane (BM). The $1 \mathrm{~mm}$ ring was defined as the center and the 3 and $6 \mathrm{~mm}$ rings as inner (1) and outer (2) rings, divided into the superior (S1, S2), nasal (N1, N2), inferior (I1, I2), and temporal (T1, T2) quadrants (Fig S1A). Average macular GCL, $\mathrm{IPL}, \mathrm{ONL}$, outer retinal, and RNFL thicknesses and volumes were automatically quantified over these same regions. One eye from one AD subject was excluded from the analyses due to poor scan quality.

Fractal Analysis. Superficial and deep vascular layers were exported in Tag Image File Format (TIFF), as automatically segmented by native Heidelberg software (Heidelberg Engineering v6.12, Heidelberg, Germany). Images were binarized using ImageJ 1.53 (National Institutes of Health, Bethesda, Maryland, USA; https://imagej.nih.gov/ij/download.html), using the local threshold Phansalkar method to a radius of 15 pixels, as previously reported, to control for variations in illuminations or contrast within the image(25). Box counting relies on the equation $N \propto \varepsilon D_{f}$, where $N$ is the number of objects; $\varepsilon$ is the linear scaling or magnification factor, which is equivalent to the inverse of the box linear dimension; and $D_{f}$ is the fractal dimension. $D_{f}$ can then be calculated by creating a $\log -\log$ plot of $N$ and $\varepsilon$ and solving for $D_{f}$, where $D_{f}=\log (N) / \log (\varepsilon)(26)$. This process is automated by the publicly available FracLac application (National Institutes of Health; http://rsb.info.nih.gov/ij/plugins/fraclac/FLHelp/Introduction.htm). 
Near Infrared Imaging. Near infrared 15deg IR ART images were exported in TIFF format from native Heidelberg software (Heidelberg Engineering v6.12, Heidelberg, Germany) and analyzed using ImageJ 1.53 (National Institutes of Health, Bethesda, Maryland, USA). The background noise was subtracted using the rolling ball method with a 50-pixel radius. The brightness/contrast of each image was then adjusted using a linear scale to saturation of vasculature and the signal intensity was measured of a specified $400 \times 400$ pixel box centered on the optic disc.

Fundus Autofluorescence Imaging. To analyze global autofluorescence, fundus autofluorescence images were exported in TIFF format from native Heidelberg software (Heidelberg Engineering v.6.12, Heidelberg, Germany) and analyzed using ImageJ 1.53 (National Institutes of Health, Bethesda, Maryland, USA). Background noise was subtracted using the rolling ball method with a 50-pixel radius. The brightness/contrast of each image was then adjusted using a linear scale to saturation of vasculature and the signal intensity was measured of a specified $430 \times 430$ pixel box in each of the following regions: supratemporal, inferotemporal, temporal, foveal, and optic nerve.

Foveal Avascular Zone. OCTA imaging was centered on the fovea and all OCTA images exported in TIFF format from native Heidelberg software (Heidelberg Engineering v.6.12, Heidelberg, Germany) were manually assessed by 3 trained double-blinded study staff. To measure foveal avascular zone (FAZ), OCTA images were thresholded using ImageJ 1.53 (National Institutes of Health, Bethesda, Maryland, USA), as previously described $(27,28)$. In brief, a polygon tool was used to encircle the FAZ. After converting to an 8-bit image, we applied thresholding to measure the FAZ area.

\section{Central Nervous System Imaging}

At a second study visit, all subjects underwent $\left[{ }^{11} \mathrm{C}\right] \mathrm{PiB}$ brain PET imaging to measure amyloid burden. PET data was acquired at the University of Michigan PET facility on a Siemens BioGraph TruePoint Model $1094 \mathrm{PET} / \mathrm{CT}$ scanner. $\left[{ }^{11} \mathrm{C}\right] \mathrm{PiB}$ imaging was obtained following a partial bolus plus constant infusion protocol ( $11.5 \mathrm{mCi}$ administered) using dynamic PET imaging of 17 time frames over 80 minutes. Reconstruction was performed using Fourier rebinning and the standard 2D-OSEM algorithm (ordered-subsets expectation maximization), using 4 iterations, 16 subsets, and 3mm Gaussian smoothing. $\left[{ }^{11} \mathrm{C}\right] \mathrm{PiB}$ was quantified as the distribution volume ratio (DVR) using the reference region Logan approach(29).Cortical DVR values were obtained using NeuroStat(30, 31), a PET neuroimaging software tool developed at the University of Michigan, and now available online. Dementia classification was confirmed with PET-amyloid measurements, and $4 / 5$ clinically diagnosed AD subjects had ${ }^{11} \mathrm{C}$-PIB values above 1.35 and were considered amyloid-positive(32). One AD subject had a PET scan that was uninterpretable due to degradation by motion.

\section{Power Calculation}

A meta-analysis(24) found a consensus effect size (standardized mean difference) of approximately - 1 when comparing RNFL between $A D$ and $C N$ control groups in 24 studies. We conducted a power analysis for a comparison of 5 subjects versus 9 subjects (our sample sizes for AD and CN subjects, respectively) 
with two eyes per subject and ICC $=0.9$. We have $80 \%$ power to detect an effect of magnitude 1.52 , and $44 \%$ power to detect an effect of magnitude 1 (all tests are two-sided with type I error controlled to alpha $=0.05)$. This implies that any negative findings in our study could be due to low power, although we are well-powered to detect effects at the upper end of the range reported in the meta-analysis.

\section{Statistical Analysis}

To assess the relationship between ocular measurements and dementia status (AD, FTD, CN), we used linear mixed effects regression, treating each ocular feature separately as an outcome, treating each eye (OD/OS) and dementia group (AD, FTD, CN) as covariates with fixed coefficients, and including random intercepts for subjects. Omnibus tests (Type III test of fixed effects) for any difference among the three dementia groups were used to quantify the evidence for the association of each ocular feature with dementia. This produced one $\mathrm{p}$-value per ocular feature, and a $\mathrm{p}$-value $<0.05$ was considered to be significant. P-values for individual comparisons were also examined. To account for multiple comparisons, we then adjusted the p-values using the Bonferroni method. Both Bonferroni corrected pvalues and uncorrected $p$-values are presented. To assess the relationship between ocular measurements and amyloid burden, we used a linear mixed effects regression, treating each ocular feature separately as an outcome, treating each eye (OD/OS) and amyloid burden as covariates with fixed coefficients, and including random intercepts for subjects. Omnibus and individual p-values were used to quantify the evidence for association of each ocular feature with amyloid burden. All statistical analyses were performed using IBM SPSS Statistics v27 and all graphs were generated using Graphpad Prism v7.0.

\section{Results}

\section{Demographics}

A total of sixteen subjects, five AD, two FTD, and nine CN controls, were enrolled in the study from December 2018 to May 2019. Table 1 includes a summary of demographics, MOCA/MMSE scores, and comorbidities within each cohort. Subjects underwent neurologic evaluation using either MOCA or MMSE testing depending upon previous neurological consultation. Hyperlipidemia was the most common comorbidity in the $A D$ cohort followed by hypertension and diabetes, whereas hypertension was the most common comorbidity in the FTD cohort. 
Table 1

Demographic characteristics.

\begin{tabular}{|llll|}
\hline Demographic & Alzheimer's Disease & Frontotemporal Dementia & Control \\
\hline Number & 5 & 2 & 9 \\
\hline Sex (M/F) & $2 / 3$ & $0 / 2$ & $1 / 8$ \\
\hline Age $( \pm$ stdev) & $67.9( \pm 3.4)$ & $65.7( \pm 4.3)$ & $64.7( \pm 5.8)$ \\
\hline MOCA ( \pm stdev) & $11.3( \pm 4.7)$ & 7 & - \\
\hline MMSE $( \pm$ stdev) & $14.5( \pm 2.1)$ & 18 & - \\
\hline Hypertension $(\%)$ & $20 \%$ & $50 \%$ & $50 \%$ \\
\hline Diabetes (\%) & $20 \%$ & $0 \%$ & $33.3 \%$ \\
\hline Hyperlipidemia (\%) & $40 \%$ & $0 \%$ & $50 \%$ \\
\hline
\end{tabular}

\section{Peripapillary RNFL thicknesses are increased in AD compared to FTD and CN eyes}

OCT was obtained to measure global as well as regional peripapillary RNFL thickness (Fig. 1A-B). Overall analysis found evidence that peripapillary RNFL thickness trended towards significance among eyes from the three groups $(C N, A D, F T D ; p=0.064)$. In initial analyses, we observed increased global peripapillary RNFL thickness in AD eyes compared to both CN $(p=0.046)$ and FTD eyes $(p=0.046)$ (Fig. 2A). AD eyes also demonstrated increased peripapillary RNFL thickness compared to $C N$ eyes in the nasal region $(p=0.043)$, as well as a trend towards significantly increased peripapillary RNFL thickness in other subregions (eg. NI) compared to both FTD and control eyes (Fig. 1B, Fig. 2B). However, none of these findings remained significant following Bonferroni correction for multiple comparisons, exhibiting corrected $p$ values $>0.05$.

\section{Peripapillary RNFL thickness is proportional to amyloid burden in $A D$ eyes}

The relationship between RNFL thickness and amyloid burden was evaluated in each dementia cohort by SD-OCT imaging. We detected a proportional relationship between global peripapillary RNFL thickness and amyloid burden in $A D$ eyes, but this relationship was not significant following Bonferroni correction $(p=0.041$, corrected $p=0.287)(F i g .3 A)$. Similarly, nasal peripapillary RNFL thickness and nasal inferior peripapillary RNFL thickness were correlated with amyloid burden in AD eyes, but the comparisons were only significant in unadjusted analyses $(p=0.037$, corrected $p=0.259$; and $p=0.05$, corrected $p=0.35$, respectively) (Fig. 3B and $3 \mathrm{C}$ ). No significant relationship was found between amyloid burden and peripapillary RNFL thickness in FTD and CN control eyes. Within the AD cohort, a trend towards decreased RNFL thickness was noted in subjects with increased amyloid burden (Fig. 3A). 


\section{Macular retinal layer thicknesses and volumes are not significantly different between dementia subtypes}

We assessed macular retinal layer thicknesses by SD-OCT in eyes from each of the cohorts. These analyses included the ganglion cell layer (GCL), the macular RNFL, the inner plexiform layer (IPL), the outer nuclear layer $(\mathrm{ONL})$, and outer retinal layer, as measured from the internal limiting membrane to the external limiting membrane. No significant differences were observed in retinal layer thicknesses among the three cohorts (Fig S2, Fig S3). We also measured retinal layer volume for macular GCL, RNFL, IPL, ONL and outer retinal layer, but likewise failed to detect significant differences in this variable between dementia subtypes (data not shown).

\section{Fractal analysis of OCTA images demonstrates decreased vascular complexity in AD eyes}

To uncover differences in the vascular complexity of the retinal vascular plexuses between dementia subtypes, we compared the fractal qualities of OCTA images within each cohort (Fig. 4A). No significant differences in vascular complexity were observed among eyes from the three dementia subtypes in the superficial vascular complex $(S V C)(p=0.067$, corrected $p=0.134)$. Fractal analysis revealed decreased vascular complexity in AD eyes compared to FTD eyes within the SVC $(p=0.035)$, but these differences were non-significant (corrected $p=.07$ ) after Bonferroni correction. No significant differences in vascular complexity were noted between dementia subtypes in the deep vascular zone (DVC; Fig. 4A), and there was no statistically significant relationship between vascular complexity and amyloid burden in $A D$ patients (Fig. 4B).

\section{Foveal avascular zone areas are not significantly different among the dementia subtypes}

Three trained, double-blinded study staff examined OCTA images and manually measured FAZ areas. No significant differences in FAZ areas were identified between the three cohorts (Fig S5).

\section{Increased fundus autofluorescence signal measurements in $A D$ eyes}

We used fundus autofluorescence imaging and near-infrared imaging to analyze the presence of dementia-specific autofluorescent proteins (eg. amyloid and lipofuscin) and retinal anatomy, respectively. Overall cohort analysis showed no significant differences in fundus autofluorescence signal intensity measurements (abu) across the cohorts $(p=0.123$, corrected $p=0.615)$ (Fig. 5). Individual comparisons of signal intensity in the inferotemporal region demonstrated an increase in fundus autofluorescence signal specifically in AD eyes compared to control eyes, but this relationship was not statistically significant upon correction for multiple comparisons $(p=0.046$, corrected $p=0.23)$. Furthermore, no 
significant differences in near-infrared reflectance were observed between dementias eyes and those from control subjects (Fig S6).

\section{Discussion}

In this study we assessed the utility of multimodal ocular imaging to identify and measure retinal biomarkers associated with AD and FTD. To our knowledge, this is amongst only a few studies to utilize SD-OCT, OCT-angiography, and fundus autofluorescence imaging to identify retinal biomarkers of neurodegeneration within a cohort of PET-confirmed amyloid-positive AD subjects, amyloid-negative FTD subjects, and amyloid-negative $\mathrm{CN}$ control subjects.

\section{Lack of retinal thinning in PET-diagnosed AD patients}

Numerous previous reports have demonstrated variable retinal layer thinning in AD and FTD eyes $(6,7$, $33,34)$. In contrast to these previous studies, we did not find any significant differences in the thicknesses of the macular RNFL, GCL, IPL, and ONL (Fig S2). Several factors could contribute to these discrepancies, including the stage of disease, diagnostic criteria, age range of subjects, and incidence of comorbidities(7). For example, glaucoma is a chronic age-related neurodegenerative disease affecting the retina that is proposed to share common mechanistic features with $A D(24)$. Additionally, most studies have been limited by small sample size and relied heavily on clinical diagnoses of dementia.

Due to the high degree of clinical overlap between dementias and rates of misdiagnosis, the diagnosis of AD should be confirmed by CSF or imaging biomarkers, such as amyloid PET imaging. In a recent metaanalysis of existing literature on RNFL changes in AD, only 8 of 25 studies included diagnostic neuroimaging (MRI or CT) and 2 studies included CSF analysis(24). Furthermore, roughly $20 \%$ of clinically diagnosed AD patients fail to show elevated amyloid burden on amyloid PET imaging, leading to false negatives(35). In our study, the diagnosis of AD was confirmed with both clinical criteria evaluated by MMSE/MOCA and PET imaging. By utilizing PET imaging, we were able to not only measure amyloid burden but also distinguish AD from other neurodegenerative disorders, such as FTD, that share similar clinical presentations and may have confounded previous studies.

\section{Peripapillary RNFL thickness is increased in PET-confirmed AD eyes}

We observed a trend towards peripapillary RNFL thickening within AD eyes compared to FTD and normal eyes. Although the majority of previous ocular imaging studies demonstrated retinal thinning within AD eyes, few studies observed thickening of the retina in $A D$ eyes $(36,37)$. Retinal thickening has been described by groups such as Snyder et al., who demonstrated volume increase in the IPL layer of the retina in preclinical $A D$ patients positive for $A \beta$ by PET imaging(13). Additionally, Snyder et al. found a positive correlation between IPL volume and the surface area of retinal inclusion bodies, thought to contain amyloid, speculating that the observed IPL thickening could represent an early inflammatory process resulting from $A \beta$ deposition(13). Similarly, the peripapillary RNFL thickening we observed was 
correlated with amyloid burden as determined by $\left[{ }^{11} \mathrm{C}\right] \mathrm{PiB}$ (Fig. 3B-C), suggesting that $A \beta$ deposition drives $A D$-associated retinal structural changes.

Although several studies identified structural, proteomic, and vascular changes in the retina of symptomatic AD subjects, many others failed to find significant differences(7). A recent investigation by den Haan et al. examined relationships between retinal thickness and established AD biomarkers (MRI, CSF analysis, amyloid-PET analysis) in AD subjects and CN controls(6). Here, retinal thickness did not differ between amyloid-positive AD cases and amyloid-negative controls in their biomarker-confirmed cohort of subjects(6). In contrast, our study suggests that peripapillary retinal thickness may be increased in amyloid-positive AD subjects compared to amyloid-negative FTD and $C N$ subjects. This discrepancy could relate to differences in inclusion criteria: our study involved subjects with moderate to severe dementia with MMSE scores < 17, while den Haan et al. focused on dementia subjects with MMSE scores $\geq 17(6)$. Increased retinal thickness may result from more severe disease. For example, increased inferior RNFL thickness is associated with lower general processing speed and lower general cognitive function, linking RNFL thickness with a higher risk of cognitive deterioration in patients with mild cognitive impairment $(30,31)$. Therefore, we speculate that in later stages of $A D$, advanced disease-related processes could contribute to peripapillary retinal thickening detectable by OCT.

The observed increase in RNFL thicknesses in our AD cohort could be explained by pathophysiological processes, such as infiltration of inflammatory cells and/or deposition of proteinaceous material within the retina(13). $A \beta$ oligomers and protein deposits may induce a potent inflammatory response, leading to increased RNFL thickness. Supporting this notion, $A D$ mouse models demonstrate deposition of $A \beta$ and tau, as well as astrogliosis within the retina $(38,39)$. Amyloid-like deposits have been visualized by OCT in the outer plexiform, ganglion and RNFL layers of the retina with the use of curcumin, a fluorescent dye that binds fibrillar amyloid(12). Similarly, we identified an increase in autofluorescence in the inferotemporal region in $A D$ subjects with a trend towards overall increased autofluorescence, suggestive of retinal amyloid protein deposition (Fig. 5).

The increased RNFL thicknesses we observed may additionally be in part due to the type of OCT employed in our study. Time domain-OCT (TD-OCT) measurements may be significantly affected by errors related to ocular parameters, such as axial length, refractive error and corneal curvature(40). We utilized the next generation of spectral domain-OCT (SD-OCT), which is the current standard of care for retinal imaging because of increased resolution, decreased acquisition time, and minimal errors related to ocular parameters. A recent meta-analysis suggests that studies showing more apparent changes in RNFL thickness in AD utilized TD-OCT rather than SD-OCT(24).

\section{OCTA analysis reveals decreased vascular complexity and normal FAZ in AD eyes}

Cerebrovascular pathology and dysfunction are increasingly described in dementias $(15,16)$. Since there is significant anatomical and physiological homology between the retinal and cerebral vasculature, it is proposed that retinal vascular changes could reflect those of the brain(16). OCTA is closely related to 
OCT and utilizes the same light capture technology to create high-resolution angiographic pictures of the retina, providing qualitative and quantitative information about the dynamics of retinal vasculature and blood flow. Our OCTA findings suggest decreased vascular complexity within AD eyes without observable change in FAZ area.

To examine vascular complexity, we utilized fractal analysis, a methodology employed to produce quantifiable fractal dimension that summarizes the complexity of a structure, with higher values indicating higher complexity(26). Fractal analysis has been utilized to study the complexity of retinal vasculature in a variety of ocular diseases including macular degeneration, retinal vein occlusion and diabetic retinopathy(41-43). Using fractal analysis, we found decreased vascular complexity in the superficial vascular complex of AD eyes compared to FTD eyes prior to adjustment for multiple comparisons. Our results support conclusions from previous studies, which have shown significantly decreased fractal dimension in $A D$ eyes compared to control eyes, and an increased probability of cognitive dysfunction in subjects with lower fractal dimension(18-20,44, 45). Interestingly, we found significant alterations in $A D$ eyes that were limited to the superficial vascular complex. There has been evidence of decreased vascular density in the deep vascular complex compared to the superficial vascular complex of the retinal vascular plexus, with decreased vessel density associated with aging(46, 47). This variability could be due in part to the difference in distribution between the complexes, as each supplies different layers within the retina. Differences in vascular density between the superficial and deep vascular complexes have also been associated with retinal disease, including diabetic retinopathy(48)

Prior OCTA studies have proposed changes in the FAZ, a retinal region susceptible to ischemia, within AD eyes $(15,16)$. In contrast, our study was unable to replicate enlargement of the FAZ in PET-confirmed AD eyes. This is similar to findings described by others, who have also been unable to detect differences in FAZ size between $A D$ eyes or eyes from preclinical $A \beta$-positive individuals and $C N$ control individuals(17, 21). All of these described studies have been limited by a relatively small number of study subjects and/or reliance on clinical diagnoses of AD. Thus, larger studies with biomarker-confirmed dementia subjects are necessary to determine whether FAZ area is altered in AD or FTD eyes.

\section{Study Limitations}

The study was limited in part due to a small sample size. Power calculation using effect sizes similar to those previously calculated by den Haan et al(24) revealed that our study had enough power to identify differences at the upper end of previously reported results (effect size -1). However, we were not powered to detect smaller differences and would thus require a larger study cohort to do so. Furthermore, as a result of the pilot study design, we performed multiple comparisons when analyzing each retinal imaging modality among the cohorts. We attempted to account for such multiple comparisons with the use of Bonferroni correction. Although we found several differences in retinal findings between the three dementia statuses in unadjusted analyses, no findings were statistically significant after Bonferroni correction. Taking into account the results from this study, additional studies are necessary to determine whether retinal markers could serve as effective biomarkers of dementia. 


\section{Conclusions}

Our study analyzed the utility of multimodal ocular imaging to identify retinal biomarkers associated with AD and FTD. Our findings, although not significant after Bonferroni correction, suggest differences in OCT and OCTA findings between AD and FTD eyes, including increased peripapillary RNFL thickness and decreased vascular complexity in $A D$ eyes. Since we were unable to reproduce the retinal thinning found in other studies, we propose that a single retinal measurement, such as retinal thickness, is not sufficient to serve as a biomarker of dementia alone. By evaluating near-infrared and fundus autofluorescence imaging, we also observed a trend towards increased fundus autofluorescence signal in AD eyes compared to $\mathrm{CN}$ control eyes. Thus, a combination of ocular imaging findings could be considered in conjunction with clinical findings to aid in diagnosis and differentiation of dementia subtype. Future studies with larger cohorts are required to establish the utility of retinal imaging measurements as biomarkers of dementia.

\section{Declarations}

\section{Ethics approval and consent to participate}

This is a case-control study approved by the Institutional Review Board at the University of Michigan. The study was registered on clinicaltrials.gov (NCT03699644) on October 9, 2018. Participants were recruited between 2018 to 2019. Written informed patient consent was received by all participants who were able to provide consent. For patients with advanced dementia, assents were obtained from the study participants and written informed consent was obtained by their legally authorized representatives.

\section{Consent for publication}

$\mathrm{N} / \mathrm{A}$

\section{Availability of data and materials}

The datasets used and/or analyzed during the current study are available from the corresponding author on reasonable request.

\section{Competing interests}

KZY reports no disclosures relevant to the manuscript; NSK reports no disclosures relevant to the manuscript; OM reports no disclosures relevant to the manuscript; BY reports no disclosures relevant to the manuscript; WP reports no disclosures relevant to the manuscript; SKS reports no disclosures relevant to the manuscript; $\mathrm{YZ}$ reports no disclosures relevant to the manuscript; KS reports no disclosures relevant to the manuscript; RK reports no disclosures relevant to the manuscript; SJB reports no disclosures relevant to the manuscript; RLA reports the following disclosures: Data Safety and Monitoring Board service for the TANGO (Biogen), SIGNAL-AD (Vaccinex), and MSTAR (Biohaven) trials, consulting 
fees from the Michael J. Fox Foundation, grant support from the Parkinson's Foundation, and P30AG053760, R21 NS114749, R01 AG058724; CGB reports no disclosures relevant to the manuscript.

\section{Funding}

Funding was provided by Multimodal ocular imaging in neurodegenerative disorders, Research Stimulus Funding Opportunity, University of Michigan Medical School, grant support from P30AG053760 (RLA), and internal funding to RLA.

\section{Author's contributions}

KZY: Drafting/revision of the manuscript for content, including medical writing for content; Analysis or interpretation of data

NK: Drafting/revision of the manuscript for content, including medical writing for content; Analysis or interpretation of data

OM: Major role in the acquisition of data; Study concept or design Benjamin Young: Analysis or interpretation of data

WP: Analysis or interpretation of data

SKS: Analysis or interpretation of data

YZ: Analysis or interpretation of data

KS: Analysis or interpretation of data

RK: Analysis or interpretation of data

SJB: Drafting/revision of the manuscript for content, including medical writing for content; Major role in the acquisition of data; Study concept or design; Analysis or interpretation of data

RLA: Drafting/revision of the manuscript for content, including medical writing for content; Major role in the acquisition of data; Study concept or design; Analysis or interpretation of data

CGB: Drafting/revision of the manuscript for content, including medical writing for content; Major role in the acquisition of data; Study concept or design; Analysis or interpretation of data

\section{Acknowledgements}

We would like to acknowledgeTimothy J. Steffens, CRA, Director of Ophthalmic Imaging and Laura Trebesh, COT at the W.K. Kellogg Eye Center, University of Michigan, in obtaining and processing the ocular imaging used in this manuscript. We would also like to acknowledge the following funding sources: NIH/NIA P30 AG053760 (SJB), NIH/NINDS R01 NS097542(SJB), R01 NS113943(SJB), and the family of Angela Dobson and Lyndon Welch (SJB). 


\section{References}

1. Moinuddin O, Khandwala NS, Young KZ, Sathrasala SK, Barmada SJ, Albin R, et al. The Role of Optical Coherence Tomography in Identifying Retinal Biomarkers in FTD: A Review. Neurology: Clinical Practice. 2021 Jan 18;:10.1212/CPJ.0000000000001041.

2. Adhi M, Duker JS. Optical coherence tomography-current and future applications. Curr Opin Ophthalmol. 2013 May;24(3):213-21.

3. Siger M, Dziegielewski K, Jasek L, Bieniek M, Nicpan A, Nawrocki J, et al. Optical coherence tomography in multiple sclerosis: thickness of the retinal nerve fiber layer as a potential measure of axonal loss and brain atrophy. J Neurol. D. Steinkopff-Verlag; 2008 Oct;255(10):1555-60.

4. Altintaş $O$, Işeri P, Ozkan B, Cağlar Y. Correlation between retinal morphological and functional findings and clinical severity in Parkinson's disease. Doc Ophthalmol. Springer-Verlag; 2008 Mar;116(2):pp. 137-46.

5. de Seze J, Blanc F, Jeanjean L, Zéphir H, Labauge P, Bouyon M, et al. Optical coherence tomography in neuromyelitis optica. Arch Neurol. American Medical Association; 2008 Jul;65(7):pp. 920-3.

6. Haan den J, van de Kreeke JA, Konijnenberg E, Kate Ten M, Braber den A, Barkhof F, et al. Retinal thickness as a potential biomarker in patients with amyloid-proven early- and late-onset Alzheimer's disease. Alzheimers Dement (Amst). John Wiley \& Sons, Ltd; 2019 Dec;11(1):pp. 463-71.

7. Alber J, Goldfarb D, Thompson LI, Arthur E, Hernandez K, Cheng D, et al. Developing retinal biomarkers for the earliest stages of Alzheimer"s disease: What we know, what we don"t, and how to move forward. Alzheimers Dement. John Wiley \& Sons, Ltd; 2020 Jan;16(1):pp. 229-43.

8. Kim BJ, Irwin DJ, Song D, Daniel E, Leveque JD, Raquib AR, et al. Optical coherence tomography identifies outer retina thinning in frontotemporal degeneration. Neurology. Wolters Kluwer Health, Inc. on behalf of the American Academy of Neurology; 2017 Oct 10;89(15):pp. 1604-11.

9. Kim BJ, Grossman M, Song D, Saludades S, Pan W, Dominguez-Perez S, et al. Persistent and Progressive Outer Retina Thinning in Frontotemporal Degeneration. Front Neurosci Frontiers. 2019;13:298.

10. Dowson JH. A sensitive method for the demonstration of senile plaques in the dementing brain. Histopathology. John Wiley \& Sons, Ltd; 1981 May;5(3):305-10.

11. Koronyo-Hamaoui M, Koronyo Y, Ljubimov AV, Miller CA, Ko MK, Black KL, et al. Identification of amyloid plaques in retinas from Alzheimer's patients and noninvasive in vivo optical imaging of retinal plaques in a mouse model. Neuroimage. 2011 Jan;54(Suppl 1):204-17.

12. Koronyo Y, Biggs D, Barron E, Boyer DS, Pearlman JA, Au WJ, et al. Retinal amyloid pathology and proof-of-concept imaging trial in Alzheimer's disease. JCI Insight. American Society for Clinical Investigation; 2017. Aug 17;2(16).

13. Snyder PJ, Johnson LN, Lim YY, Santos CY, Alber J, Maruff P, et al. Nonvascular retinal imaging markers of preclinical Alzheimer's disease. Alzheimers Dement (Amst). John Wiley \& Sons, Ltd; 2016;4(1):pp. 169-78. 
14. Ward ME, Chen R, Huang H-Y, Ludwig C, Telpoukhovskaia M, Taubes A, et al. Individuals with progranulin haploinsufficiency exhibit features of neuronal ceroid lipofuscinosis. Sci Transl Med. American Association for the Advancement of Science; 2017 Apr 12;9(385).

15. Bulut M, Kurtuluş F, Gözkaya O, Erol MK, Cengiz A, Akıdan M, et al. Evaluation of optical coherence tomography angiographic findings in Alzheimer's type dementia. $\mathrm{Br} \mathrm{J}$ Ophthalmol. BMJ Publishing Group Ltd; 2018 Feb;102(2):233-7.

16. O'Bryhim BE, Apte RS, Kung N, Coble D, Van Stavern GP. Association of Preclinical Alzheimer Disease With Optical Coherence Tomographic Angiography Findings. JAMA Ophthalmol. American Medical Association; 2018 Nov 1;136(11):pp. 1242-8.

17. Yoon SP, Grewal DS, Thompson AC, Polascik BW, Dunn C, Burke JR, et al. Retinal Microvascular and Neurodegenerative Changes in Alzheimer's Disease and Mild Cognitive Impairment Compared with Control Participants. Ophthalmol Retina. 2019 Jun;3(6):489-99.

18. Lemmens S, Devulder A, Van Keer K, Bierkens J, De Boever P, Stalmans I. Systematic Review on Fractal Dimension of the Retinal Vasculature in Neurodegeneration and Stroke: Assessment of a Potential Biomarker. Front Neurosci Frontiers. 2020;14:16.

19. Cabrera DeBuc D, Somfai GM, Arthur E, Kostic M, Oropesa S, Mendoza Santiesteban C. Investigating Multimodal Diagnostic Eye Biomarkers of Cognitive Impairment by Measuring Vascular and Neurogenic Changes in the Retina. Front Physiol Frontiers. 2018;9:1721.

20. Williams MA, McGowan AJ, Cardwell CR, Cheung CY, Craig D, Passmore P, et al. Retinal microvascular network attenuation in Alzheimer's disease. Alzheimers Dement (Amst). John Wiley \& Sons, Ltd; 2015 Jun;1(2):pp. 229-35.

21. van de Kreeke JA, Nguyen H-T, Konijnenberg E, Tomassen J, Braber den A, Kate Ten M, et al. Optical coherence tomography angiography in preclinical Alzheimer's disease. $\mathrm{Br} \mathrm{J}$ Ophthalmol. BMJ Publishing Group Ltd; 2020 Feb;104(2):157-61.

22. Jiang $H$, Liu $Y$, Wei $Y$, Shi $Y$, Wright $C B$, Sun $X$, et al. Impaired retinal microcirculation in patients with Alzheimer's disease. PLOS ONE Public Library of Science. 2018;13(2):e0192154.

23. Feke GT, Hyman BT, Stern RA, Pasquale LR. Retinal blood flow in mild cognitive impairment and Alzheimer's disease. Alzheimers Dement (Amst). John Wiley \& Sons, Ltd; 2015 Jun;1(2):pp. 144-51.

24. Haan den J, Verbraak FD, Visser PJ, Bouwman FH. Retinal thickness in Alzheimer's disease: A systematic review and meta-analysis. Alzheimers Dement (Amst). John Wiley \& Sons, Ltd; 2017;6(1):pp. 162-70.

25. Spaide RF. Choriocapillaris Flow Features Follow a Power Law Distribution: Implications for Characterization and Mechanisms of Disease Progression. Am J Ophthalmol. 2016 Oct;170:58-67.

26. Mandelbrot BB. The Fractal Geometry of Nature. Times Books; 1983. 1 p.

27. Shiihara H, Terasaki H, Sonoda S, Kakiuchi N, Shinohara Y, Tomita M, et al. Objective evaluation of size and shape of superficial foveal avascular zone in normal subjects by optical coherence tomography angiography. Sci Rep Nature Publishing Group. 2018 Jul;8(1)(4):10143-9. 
28. Zdilla MJ, Hatfield SA, McLean KA, Cyrus LM, Laslo JM, Lambert HW. Circularity. Solidity, Axes of a Best Fit Ellipse, Aspect Ratio, and Roundness of the Foramen Ovale: A Morphometric Analysis With Neurosurgical Considerations. J Craniofac Surg. 2016 Jan;27(1):222-8.

29. Logan J, Fowler JS, Volkow ND, Wang GJ, Ding YS, Alexoff DL. Distribution volume ratios without blood sampling from graphical analysis of PET data. J Cereb Blood Flow Metab. SAGE PublicationsSage UK: London; 1996 Sep;16(5):pp. 834-40.

30. Shen Y, Shi Z, Jia R, Zhu Y, Cheng Y, Feng W, et al. The attenuation of retinal nerve fiber layer thickness and cognitive deterioration. Front Cell Neurosci Frontiers. 2013;7:142.

31. Laude A, Lascaratos G, Henderson RD, Starr JM, Deary IJ, Dhillon B. Retinal nerve fiber layer thickness and cognitive ability in older people: the Lothian Birth Cohort 1936 study. BMC Ophthalmol BioMed Central. 2013 Jul;13(1)(3):28-7.

32. Liu E, Schmidt ME, Margolin R, Sperling R, Koeppe R, Mason NS, et al. Amyloid- $\beta$ 11C-PiB-PET imaging results from 2 randomized bapineuzumab phase 3 AD trials. Neurology. Wolters Kluwer Health, Inc. on behalf of the American Academy of Neurology; 2015 Aug 25;85(8):692-700.

33. Golzan SM, Goozee K, Georgevsky D, Avolio A, Chatterjee P, Shen K, et al. Retinal vascular and structural changes are associated with amyloid burden in the elderly: ophthalmic biomarkers of preclinical Alzheimer's disease. Alzheimers Res Ther. BioMed Central; 2017 Mar 1;9(1):13-9.

34. Haan den J, Csinscik L, Parker T, Paterson RW, Slattery CF, Foulkes A, et al. Retinal thickness as potential biomarker in posterior cortical atrophy and typical Alzheimer's disease. Alzheimers Res Ther. BioMed Central; 2019 Jul 18;11(1):62-9.

35. Ossenkoppele R, Jansen WJ, Rabinovici GD, Knol DL, van der Flier WM, van Berckel BNM, et al. Prevalence of amyloid PET positivity in dementia syndromes: a meta-analysis. JAMA. American Medical Association; 2015 May 19;313(19):1939-49.

36. Salobrar-Garcia E, Hoyas I, Leal M, de Hoz R, Rojas B, Ramirez Al, et al. Analysis of Retinal Peripapillary Segmentation in Early Alzheimer's Disease Patients. Biomed Res Int Hindawi. 2015;2015:636548.

37. Jáñez-Escalada L, Jáñez-García L, Salobrar-Garcia E, Santos-Mayo A, de Hoz R, Yubero R, et al. Spatial analysis of thickness changes in ten retinal layers of Alzheimer's disease patients based on optical coherence tomography. Sci Rep Nature Publishing Group. 2019 Sep;10(1):13000-14. 9(.

38. Dong Z-Z, Li J, Gan Y-F, Sun X-R, Leng Y-X, Ge J. Amyloid beta deposition related retinal pigment epithelium cell impairment and subretinal microglia activation in aged APPswePS1 transgenic mice. Int J Ophthalmol. 2018;11(5):747-55.

39. Grimaldi A, Brighi C, Peruzzi G, Ragozzino D, Bonanni V, Limatola C, et al. Inflammation, neurodegeneration and protein aggregation in the retina as ocular biomarkers for Alzheimer's disease in the 3xTg-AD mouse model. Cell Death Dis. Nature Publishing Group; 2018 Jun 7;9(6):685-10.

40. Gharbiya M, Trebbastoni A, Parisi F, Manganiello S, Cruciani F, D'Antonio F, et al. Choroidal thinning as a new finding in Alzheimer's disease: evidence from enhanced depth imaging spectral domain optical coherence tomography. J Alzheimers Dis IOS Press. 2014;40(4):907-17. 
41. Cheung N, Donaghue KC, Liew G, Rogers SL, Wang JJ, Lim S-W, et al. Quantitative assessment of early diabetic retinopathy using fractal analysis. Diabetes Care American Diabetes Association. 2009 Jan;32(1):106-10.

42. Young BK, Kovacs KD, Adelman RA. Fractal Dimension Analysis of Widefield Choroidal Vasculature as Predictor of Stage of Macular Degeneration. Transl Vis Sci Technol. The Association for Research in Vision and Ophthalmology; 2020 Jun;9(7):22-2.

43. Kashani AH, Lee SY, Moshfeghi A, Durbin MK, Puliafito CA. OPTICAL COHERENCE TOMOGRAPHY ANGIOGRAPHY OF RETINAL VENOUS OCCLUSION. Retina. 2015 Nov;35(11):2323-31.

44. Csincsik L, MacGillivray TJ, Flynn E, Pellegrini E, Papanastasiou G, Barzegar-Befroei N, et al. Peripheral Retinal Imaging Biomarkers for Alzheimer's Disease: A Pilot Study. Ophthalmic Res Karger Publishers. 2018;59(4):182-92.

45. Cheung CY-L, Ong S, Ikram MK, Ong YT, Chen CP, Venketasubramanian N, et al. Retinal vascular fractal dimension is associated with cognitive dysfunction. J Stroke Cerebrovasc Dis. 2014 Jan;23(1):43-50.

46. Campbell JP, Zhang M, Hwang TS, Bailey ST, Wilson DJ, Jia Y, et al. Detailed Vascular Anatomy of the Human Retina by Projection-Resolved Optical Coherence Tomography Angiography. Sci Rep Nature Publishing Group. 2017 Feb;10(1):42201-11. 7(.

47. Lavia C, Bonnin S, Maule M, Erginay A, Tadayoni R, Gaudric A. VESSEL DENSITY OF SUPERFICIAL, INTERMEDIATE, AND DEEP CAPILLARY PLEXUSES USING OPTICAL COHERENCE TOMOGRAPHY ANGIOGRAPHY. Retina. 2019 Feb;39(2):247-58.

48. Samara WA, Shahlaee A, Adam MK, Khan MA, Chiang A, Maguire JI, et al. Quantification of Diabetic Macular Ischemia Using Optical Coherence Tomography Angiography and Its Relationship with Visual Acuity. Ophthalmology. 2017 Feb;124(2):235-44.

\section{Figures}



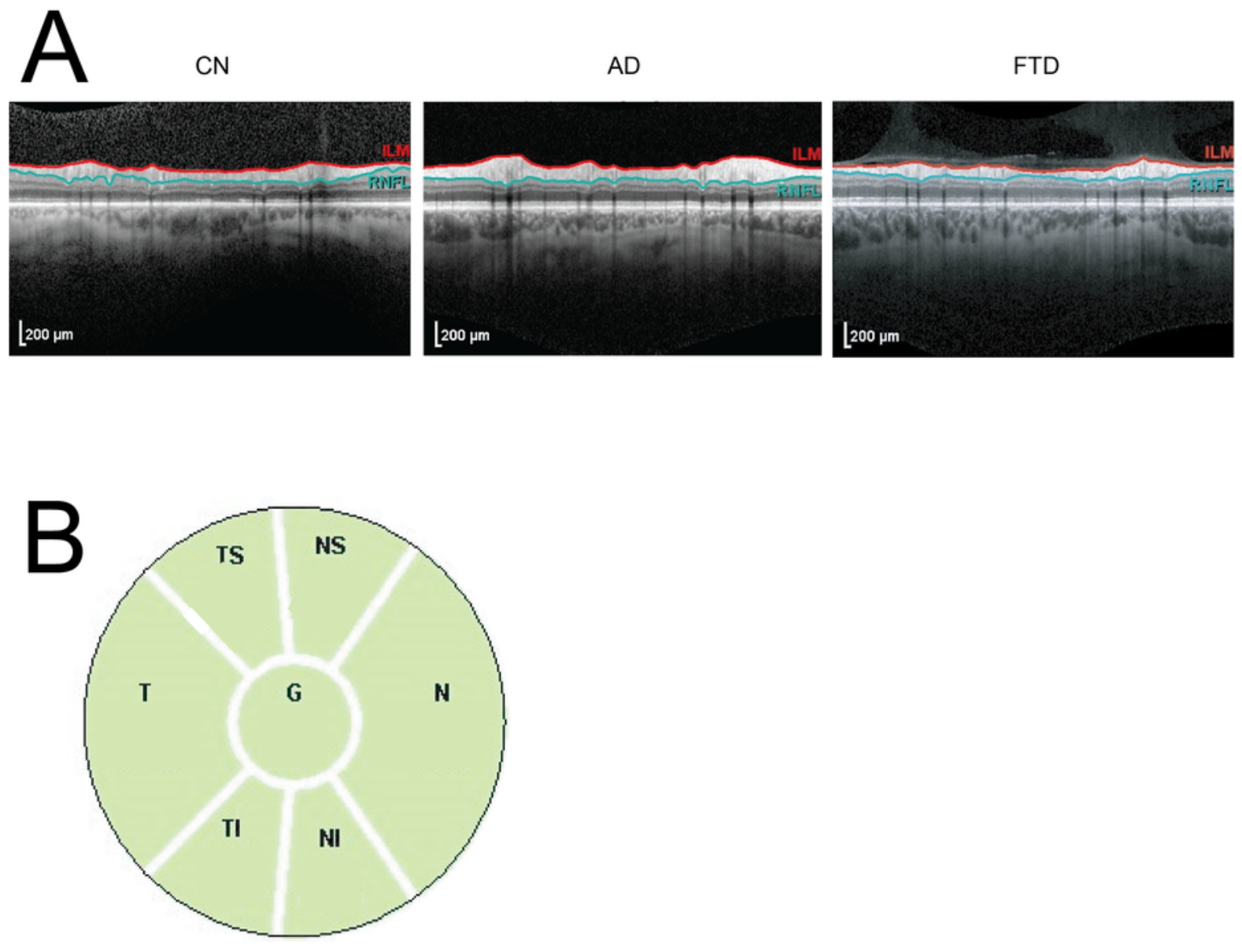

\section{Figure 1}

\section{Figure 1}

Optical coherence tomography imaging of the retinal nerve fiber layer. A. Schematic image depicting anatomical position of the nerve fiber layer within the retina in CN, AD and FTD subjects. RNFL, retinal nerve fiber layer; ILM, internal limiting membrane $B$. Anatomic delineation of regions of the retinal nerve fiber layer within $3.5 \mathrm{~mm}$ diameter circular area of the retina. $\mathrm{CN}$, cognitively normal; AD, Alzheimer's 
dementia; FTD, Frontotemporal dementia; G, global; N, nasal; NI, nasal inferior; TI, temporal inferior; T, temporal; TS, temporal superior; NS, nasal superior.

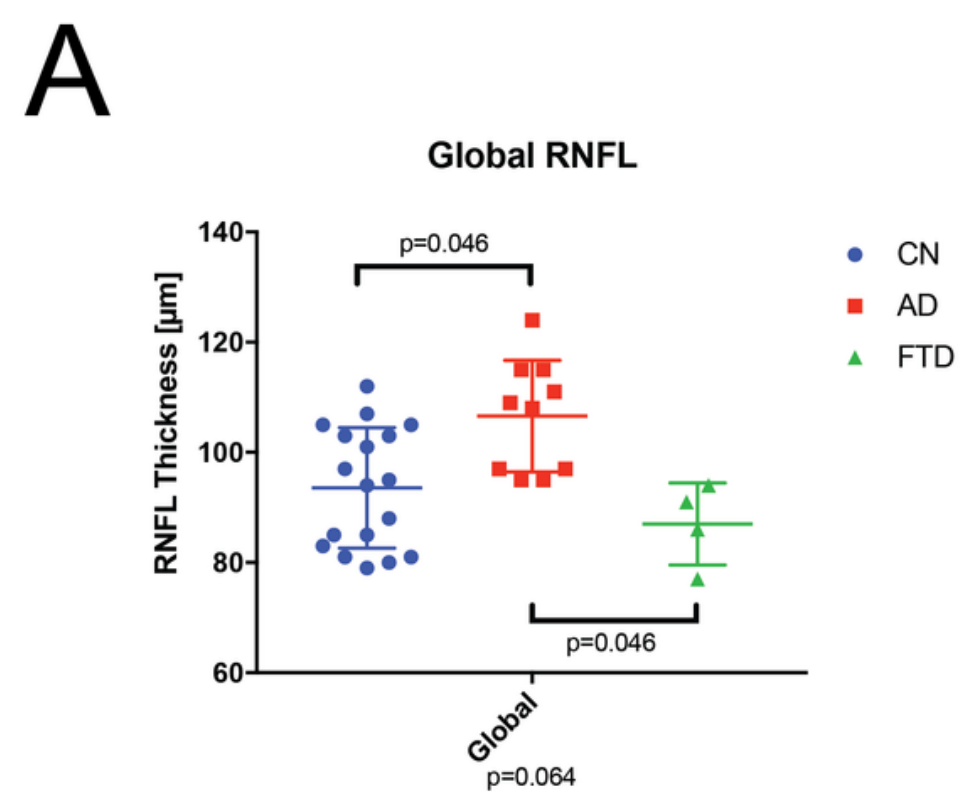

B

RNFL Subregion

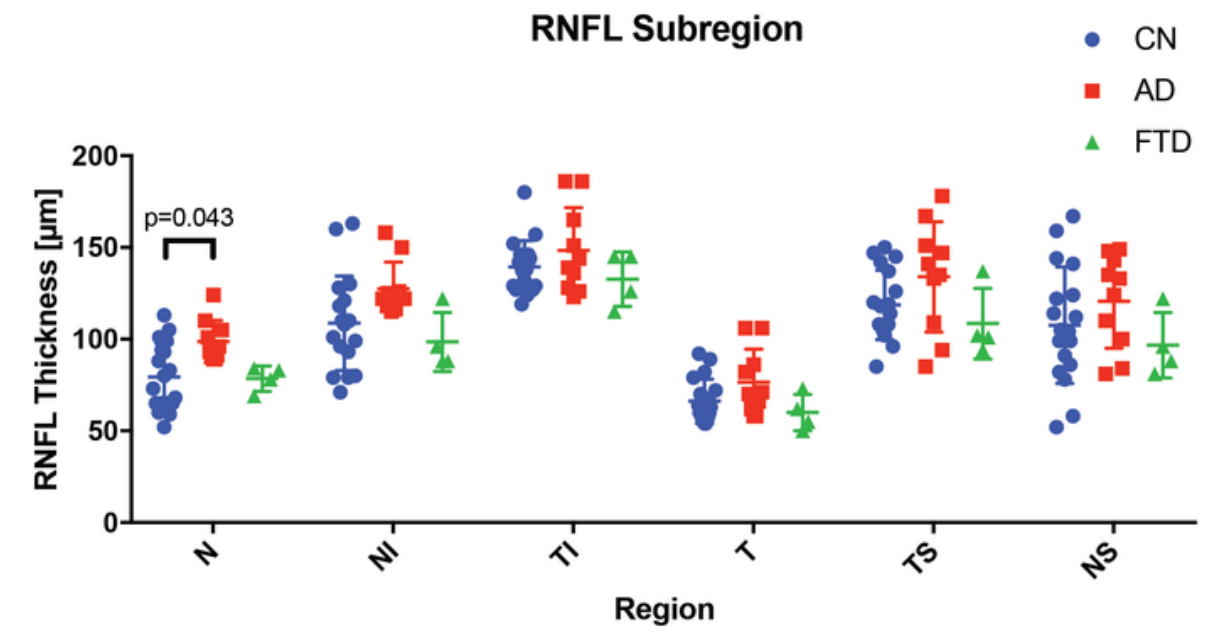

\section{Figure 2}

\section{Figure 2}

Retinal nerve fiber layer thickness increased in Alzheimer's subjects. A. Global average RNFL thickness among dementia subtypes. Alzheimer's eyes demonstrate a trend towards increased global RNFL thickness compared to CN eyes (uncorrected $p=0.046$ ) and FTD eyes (uncorrected $p=0.046$ ). B. RNFL 
thickness divided by retinal subregion (see Figure 1B). AD eyes demonstrate increased RNFL thickness compared to $\mathrm{CN}$ eyes in the nasal region (uncorrected $p=0.043$ ). AD subjects demonstrate a trend towards increased RNFL thickness among other subregions compared to CN and FTD eyes. Findings were not significant following Bonferroni correction. RNFL, retinal nerve fiber layer; $\mathrm{CN}$, cognitively normal; $A D$, Alzheimer's dementia; FTD, Frontotemporal dementia; N, nasal; NI, nasal inferior; TI, temporal inferior; T, temporal; TS, temporal superior; NS, nasal superior.

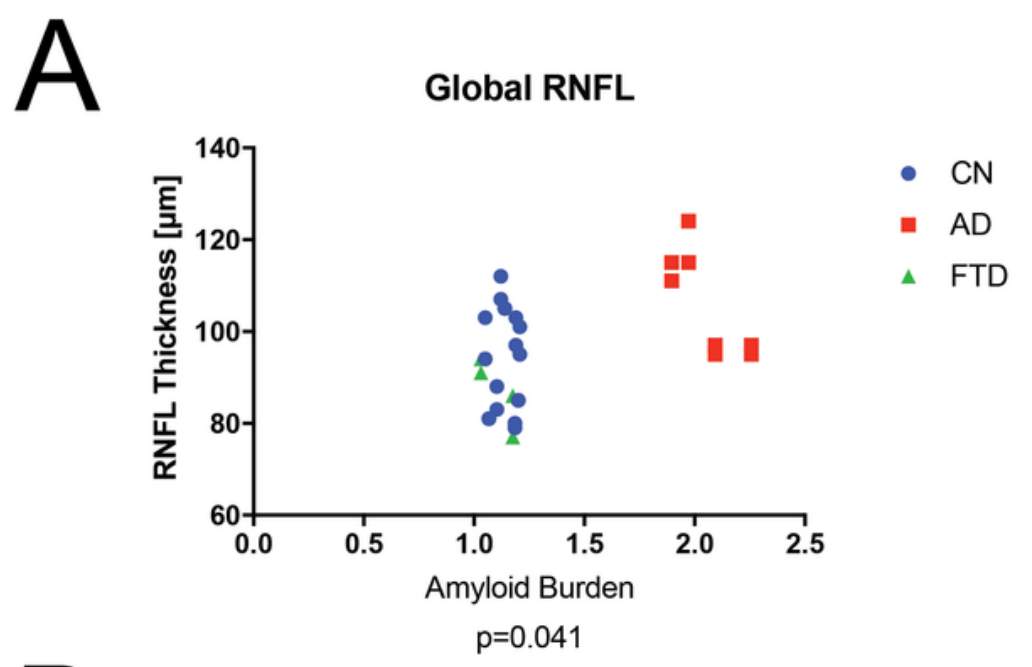

B

RNFL N Subregion

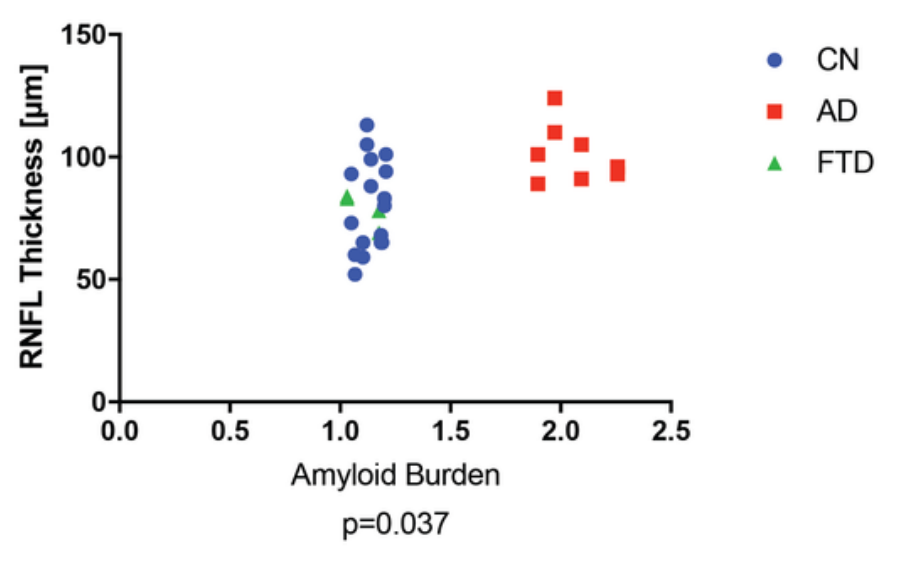

$\mathrm{C}$

RNFL NI Subregion

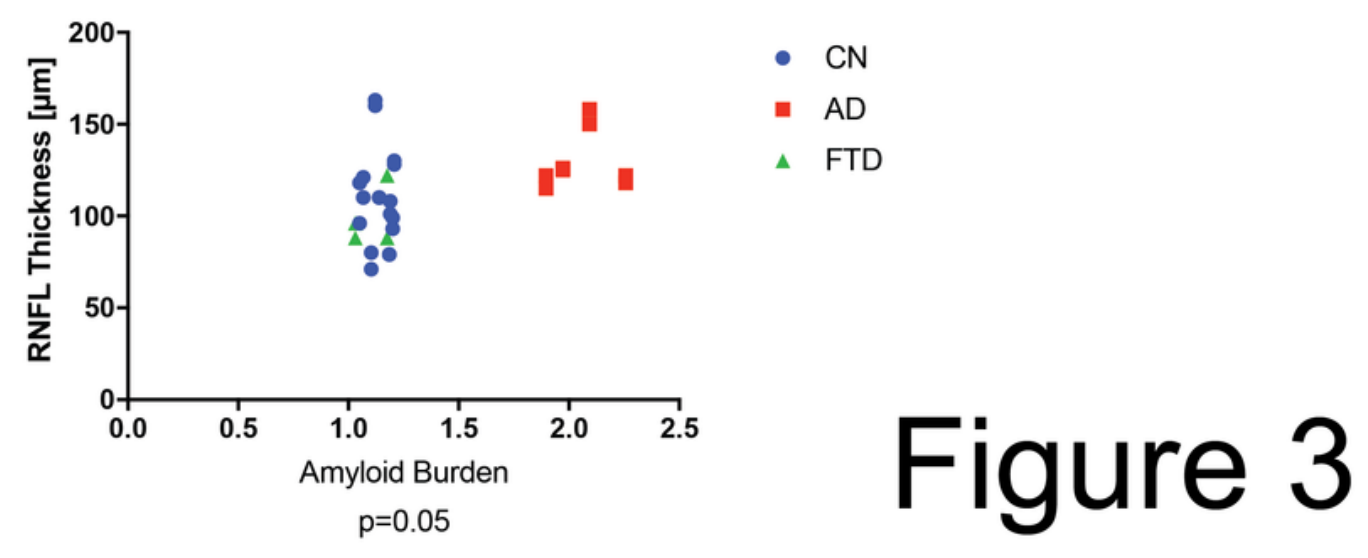

Figure 3 
Retinal nerve fiber layer thickness correlates with amyloid burden. A. Global RNFL thickness measurements positively correlate with amyloid burden in AD eyes (uncorrected $p=0.041$ ). B. Nasal RNFL thickness measurements positively correlate with amyloid burden in AD eyes (uncorrected $p=0.037$ ). $C$. Nasal inferior RNFL thickness measurements positively correlate with amyloid burden in AD eyes (uncorrected $\mathrm{p}=0.050$ ). Findings were not significant following Bonferroni correction. $\mathrm{CN}$, cognitively normal; AD, Alzheimer's dementia; FTD, Frontotemporal dementia; N, nasal; NI, nasal inferior.

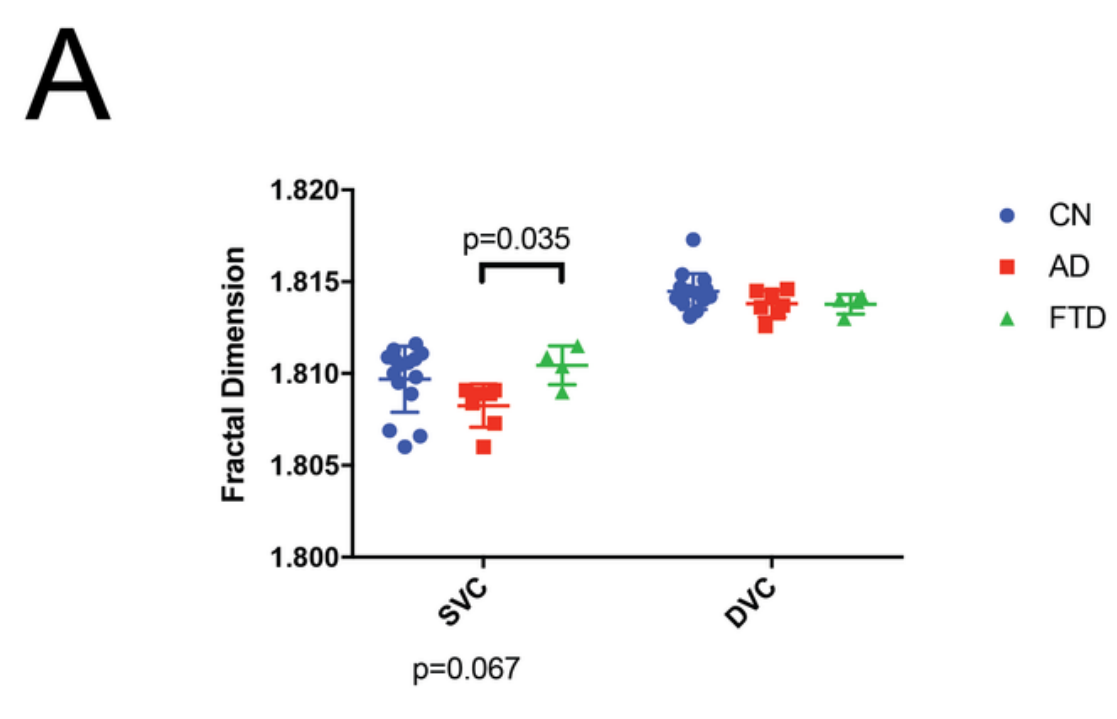

B

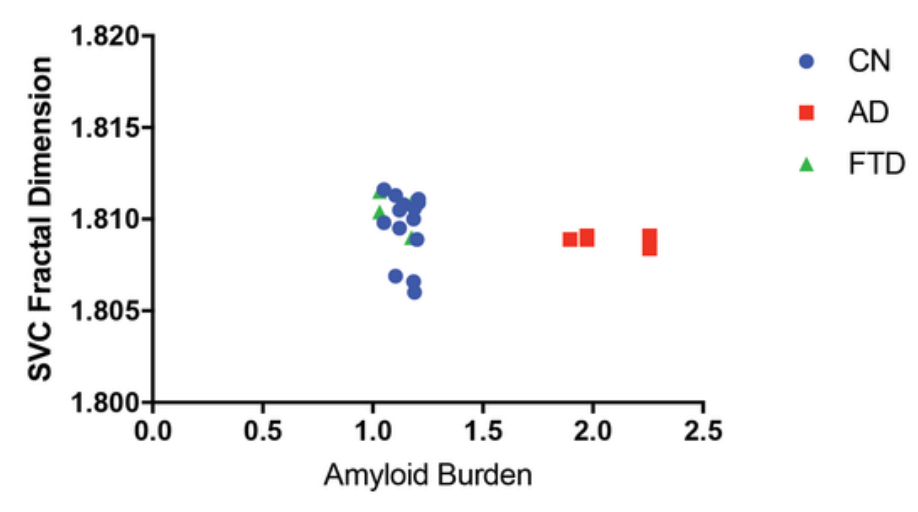

Figure 4

Figure 4 
Fractal analysis demonstrates variability in vascular complexity among dementia subtypes on OCTangiography. A. Fractal analysis demonstrates significant differences in fractal dimension between $A D$ and FTD eyes in the superficial vascular complex (SVC) of the retinal vascular plexuses (uncorrected $\mathrm{p}=$ 0.035 ) but not the deep vascular complex (DVC). Fractal dimension is not associated with dementia subtype in the deep vascular complex of the foveal avascular zone. B. Fractal analysis of the superficial vascular complex of the foveal avascular zone is not associated with cerebral amyloid burden. Findings were not significant following Bonferroni correction. $C N$, cognitively normal; $A D$, Alzheimer's dementia; DVC, deep vascular complex; FTD, frontotemporal dementia; SVC, superficial vascular complex. 

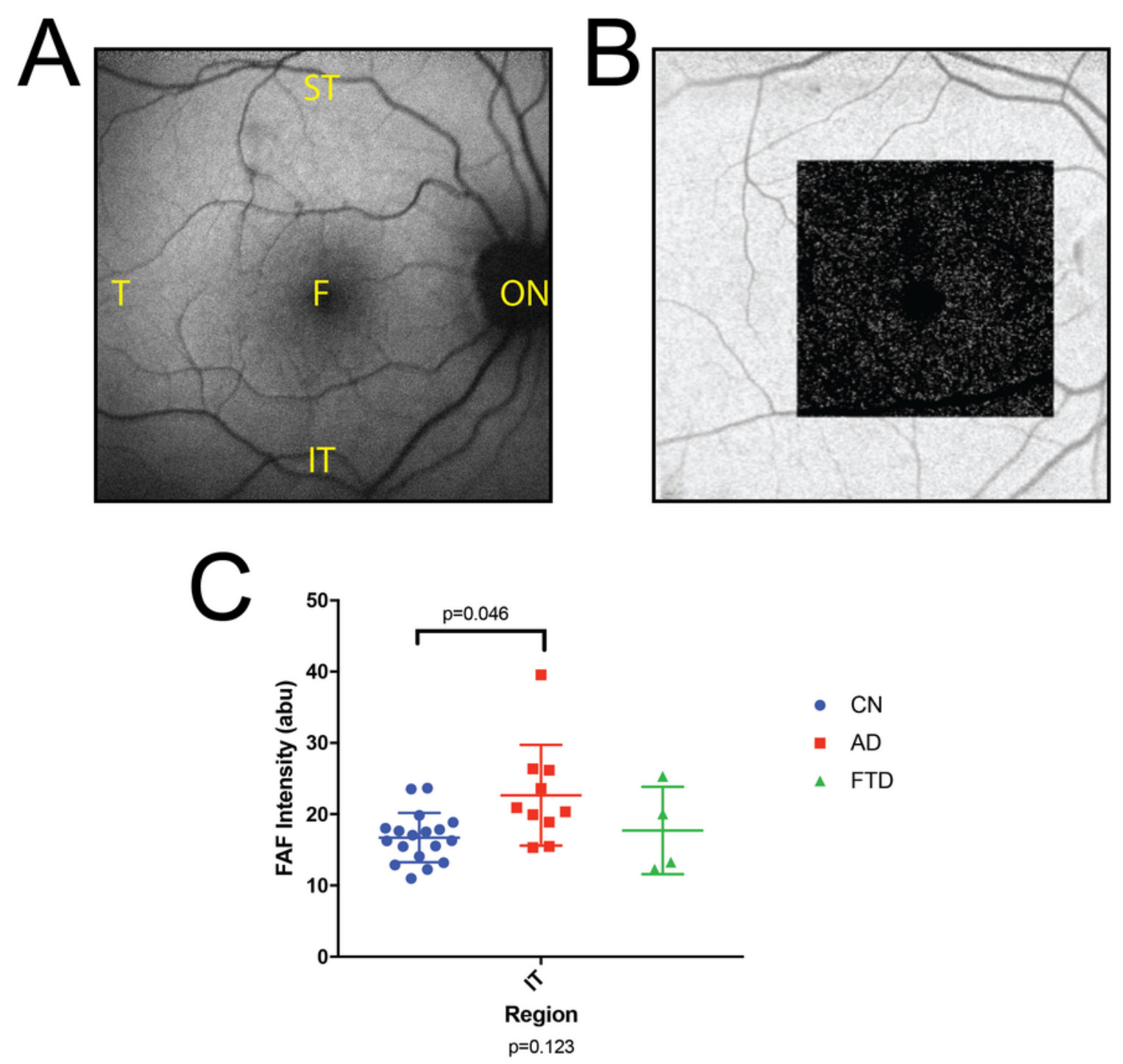

Figure 5

\section{Figure 5}

Fundus autofluorescence is associated with dementia subtype in the inferotemporal region. A. Fundus autofluorescence image from a cognitively normal subject centered around the fovea. Further analysis was done in the other anatomic regions of the retina. B. Fundus autofluorescence image processed using ImageJ to evaluate for signal intensity within standardized area of the foveal region (see methods section). C. Signal intensity of fundus autofluorescence differs between AD and CN eyes in the 
inferotemporal region of the retina (uncorrected $p=0.046$, group $p=0.123$ ). Findings were not significant following Bonferroni correction. CN, cognitively normal; AD, Alzheimer's dementia; FTD, Frontotemporal dementia; F, fovea; ST; supratemporal; ON, optic nerve; IT, infratemporal; T, temporal.

\section{Supplementary Files}

This is a list of supplementary files associated with this preprint. Click to download.

- 8.5.21Supplementallnformation.docx 